\title{
Evaluación del comportamiento mecánico de compuestos PEI - fibra de vidrio ante solicitaciones térmicas
}

\author{
L. CHACÓN'1, J. VIÑA², A. ARGÜELLES², R. ZENASNI', I. VIÑA ${ }^{2}$ \\ 'Escuela de Ingeniería Metalúrgica y Ciencia de los Materiales, Universidad Central de Venezuela \\ ${ }^{2}$ Escuela Politécnica Superior de Ingeniería, Universidad de Oviedo, Campus de Viesques, 33204 Gijón \\ ${ }^{3}$ Departamento de Física, Universidad de Ciencias y de Tecnología de Orán (Argelia)
}

\begin{abstract}
Se ha estudiado el comportamiento mecánico de un compuesto fabricado con un termoplástico PEI (polieterimida) reforzado con tejido de fibra de vidrio, frente a ensayos en los que se mantienen tensiones iguales a un tercio de su resistencia a tracción a 50 , 100 y $150{ }^{\circ} \mathrm{C}$, durante periodos comprendidos entre 250 y 3000 minutos. En los resultados se observa una disminución de la resistencia y de la deformación en las tres temperaturas, con un mantenimiento de sus valores hasta las ensayadas a 3000 minutos. Además fueron expuestas un grupo de probetas del material, a envejecimiento acelerado en cámara higrotérmica a $70{ }^{\circ} \mathrm{C}$ y a un $95 \%$ de H.R., durante 30 días; para luego someterlas a los ensayos antes descritos. Para esas probetas se obtuvieron pequeñas fluctuaciones de la resistencia y deformación con el tiempo, sin observarse una influencia significativa de la temperatura a la que se hicieron los ensayos termomecánicos.
\end{abstract}

Palabras clave: PEI, fibra de vidrio, envejecimiento higrotérmico, resistencia a tracción.

\section{Evaluation of the mechanical behavior of composite PEI - glass fiber subjected to thermal solicitations}

The mechanical behavior of a composite with thermoplastic matrix and glass fiber reinforcement has been studied. It has been tested to constant stresses of a value equivalent to tensile strength divided by three to 50,100 and $150{ }^{\circ} \mathrm{C}$, during periods of time between 250 and 3000 minutes. A decrement of the tensile strength and deformation can be observed in the three temperatures. Also a group of specimens have been exposed to accelerated aging in an environmental chamber to $70{ }^{\circ} \mathrm{C}$ and $95 \% \mathrm{RH}$, during 30 days; and after they have been tested. In that case a decrement of the strength and deformation after 250 minutes has been observed, but in larger time of exposure these properties suffered an increment.

Keywords: PEI, glass fiber, aging, tensile strength.

\section{INTRODUCCIÓN}

Los materiales compuestos formados de resinas termoplásticas reforzadas con fibra de vidrio son muy empleados en el sector aeronáutico, debido a su rigidez, resistencia a la corrosión y elevadas resistencias específicas y a fatiga. Sin embargo, a estos materiales se les ha negado su uso en ambientes con temperaturas superiores a la medioambiental, debido al habitual bajo valor de su temperatura de transición vítrea. El motivo antes descrito ha propiciado que en la bibliografía existente se han hallado pocos trabajos que estudien el comportamiento termomecánico de compuestos con refuerzo en forma de tejido $(1,2,3)$. Por otra parte, cuando se somete este material, a ambientes con altas concentraciones de humedad, durante periodos más o menos prolongados, se produce en él una progresiva absorción de agua, que depende de variables como: el tipo de fibra y de matriz, la temperatura, etc. En la práctica, la presencia de alta concentración de humedad ocurre a temperaturas relativamente elevadas, lo que hace que sea casi obligatorio el estudio conjunto de esos dos parámetros; o sea temperatura y humedad (4). Parece demostrado que la humedad absorbida por el material compuesto depende de la estructura química de la matriz, de la temperatura y de la humedad relativa, lo cual produce una plastificación tanto en la matriz como en el refuerzo, así como una alteración dimensional que induce modificaciones en las propiedades mecánicas $(5,6)$.En este trabajo se busca estudiar el comportamiento de probetas de un material compuesto fabricado con una matriz termoplástica PEI y reforzado de fibra de vidrio, frente a ensayos térmicos con tensiones fijas iguales a la tercera parte de la carga de rotura, a tres temperaturas prefijadas, inferiores a la de transición vítrea del material, durante distintos periodos de tiempo de mantenimiento entre 250 y 3000 minutos. Además, se evalúa el comportamiento de un grupo de probetas sometidas previamente a envejecimiento acelerado durante treinta días en una cámara higrotérmica, y luego a los mismos ensayos a los que se someten las no envejecidas.

\section{PARTE EXPERIMENTAL}

Este trabajo se ha realizado con un compuesto fabricado por Ten Cate Advanced Composites, formado por una matriz termoplástica (polieterimida), reforzada con tejido de fibra de vidrio, con una disposición de fibras del tipo 8 Harness Satin y una fracción volumétrica de fibras del $67 \%$.

En la figura 1 se representa un esquema de la probeta elaborada para la realización de todos los ensayos, siguiendo la norma ISO/DIS 3268.

Con las probetas mecanizadas, se realizaron varios ensayos previos de tracción a distintas temperaturas, entre la ambiental y la de transición vítrea del material $\left(\mathrm{Tg} \approx 200^{\circ} \mathrm{C}\right)$, en una máquina de tracción Instron, a la que se le acopló una cámara térmica, determinándose así las temperaturas de los posteriores ensayos de mantenimiento térmico y sus valores de resistencia en cada temperatura. A cada una de las tres temperaturas prefijadas y con tensión fija igual a un tercio del valor de la resistencia a tracción, se realizaron una serie de ensayos 


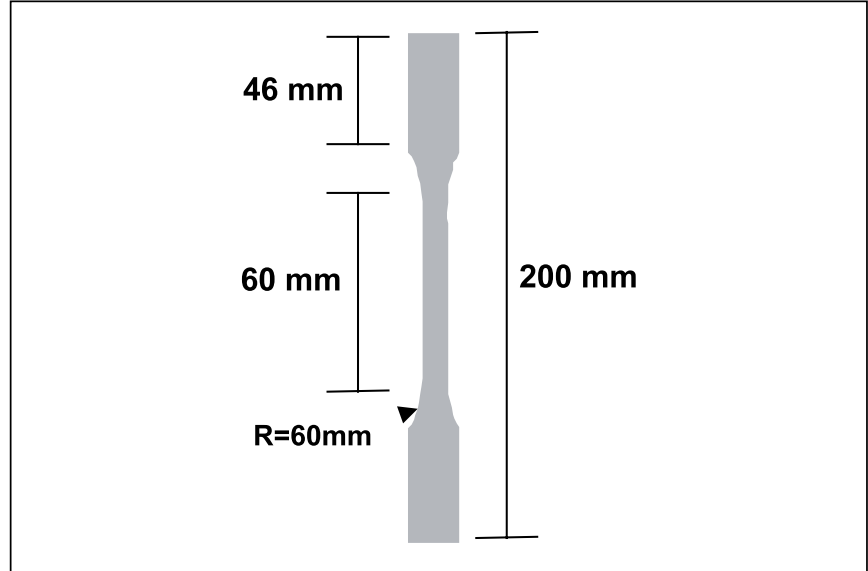

Figura 1: Esquema de las probetas ensayadas

termomecánicos con tiempos de 250, 500, 750, 1000, 2000 y 3000 minutos, ensayando hasta tres probetas en cada periodo. Paralelamente, un grupo de probetas fueron sometidas a envejecimiento acelerado en una cámara higrotérmica a $70^{\circ} \mathrm{C}$ y $95 \%$ de H.R., durante un periodo de 30 días. Cumplido ese tiempo, cada probeta envejecida se sometió a un ensayo termomecánico, siguiendo el procedimiento antes descrito. Después de cada uno de los ensayos, se realizó a cada probeta, tanto a las envejecidas como a las no expuestas, un ensayo de tracción para determinar su resistencia y deformación máximas a la rotura.

\section{RESULTADOS Y DISCUSIÓN}

\subsection{Ensayos previos}

En la figura 2 se representan las resistencias, obtenidas en un grupo de cinco probetas, para cada una de las temperaturas ensayadas a tracción.

A partir de la fig. 2 se seleccionaron como temperaturas para la realización de los posteriores ensayos, las de 50, 100 y $150{ }^{\circ} \mathrm{C}$, por encontrarse casi equidistantes sus valores de resistencia máxima de rotura. A esas tres temperaturas les correspondieron, respectivamente, como resistencias medias de rotura: 406, 344 y $285 \mathrm{MPa}$. Un tercio de cada una de esas tres tensiones es la que se empleó como valor fijo para cada temperatura en los ensayos termomecánicos.

\subsection{Ensayos termomecánicos a probetas no envejecidas}

En la fig. 3 se representan dos gráficos con los valores máximo, mínimo y medio de la resistencia y de la deformación máxima a rotura, obtenidos al ensayar a tracción un grupo de tres probetas no envejecidas que habían sido sometidas a pruebas termomecánicas en cada tiempo de ensayo para cada una de las tres temperaturas empleadas.

Se observa que todos los valores medios de la resistencias a la tracción a $50{ }^{\circ} \mathrm{C}$ son menores al de las probetas sin someter a los ensayos termomecánicos (433,06 MPa), fluctuando entre $385 \mathrm{MPa}$ y $415 \mathrm{MPa}$; los obtenidos en los ensayos a $100{ }^{\circ} \mathrm{C}$ sólo son mínimamente superiores para un tiempo mantenimiento de 500 minutos $(0,12 \%)$; y los de $150^{\circ} \mathrm{C}$ lo son en 250 minutos $(1,7 \%)$, por lo que se podría decir que la resistencia a la tracción disminuye por el efecto de la combinación esfuerzo constante-temperatura. Por otra parte no parece existir influencia de la temperatura sobre la evolución de la resistencia. En cuanto

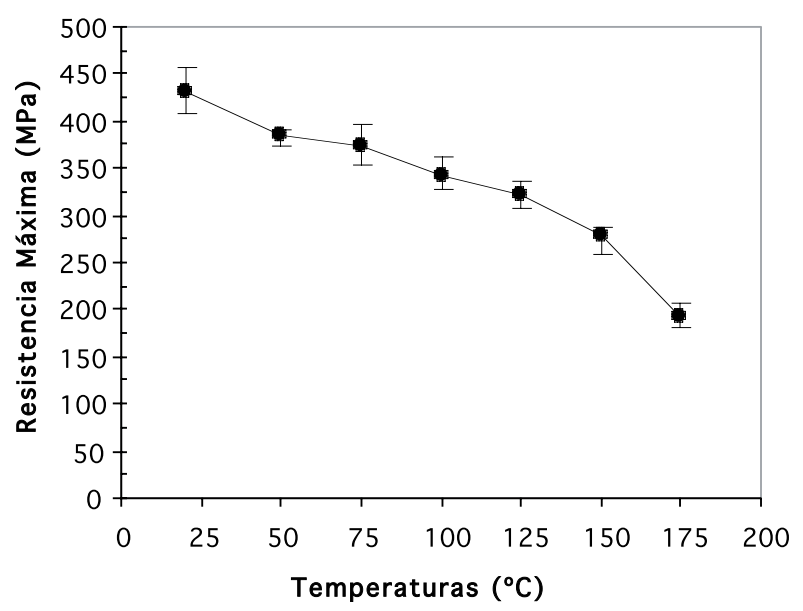

Figura 2: Resistencia vs. Temperatura
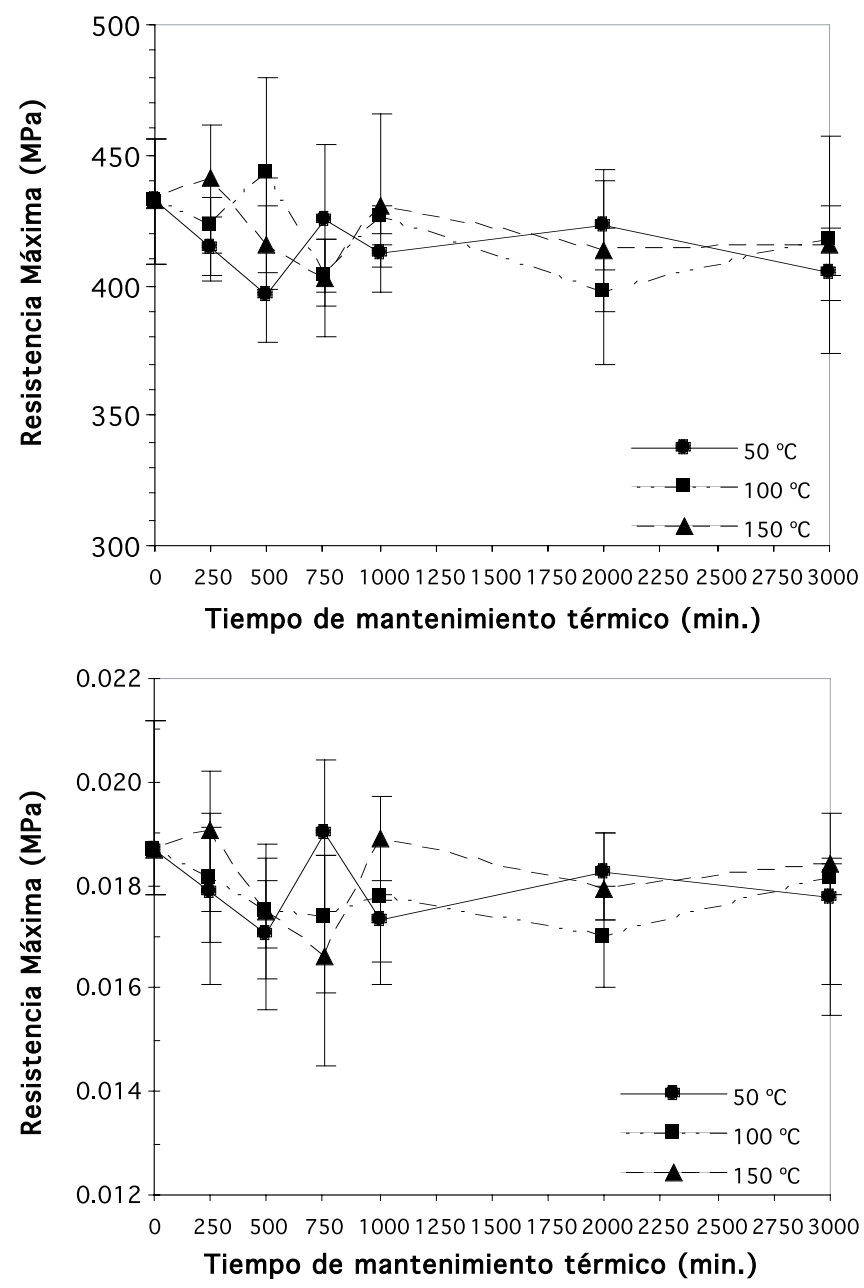

Figura 3: Valores medios, máximos y mínimos de la resistencia y la deformación en función del tiempo en los ensayos efectuados a probetas sin envejecer

a lo obtenido con las deformaciones de las probetas, los resultados siguen una tendencia análoga a las resistencias.

En ambos gráficos no se aprecia un cambio significativo en el comportamiento de las curvas en los tiempos de mantenimiento entre 2000 y 3000 minutos, por lo que en ensayos posteriores sólo alcanzaron los 2000 minutos. 

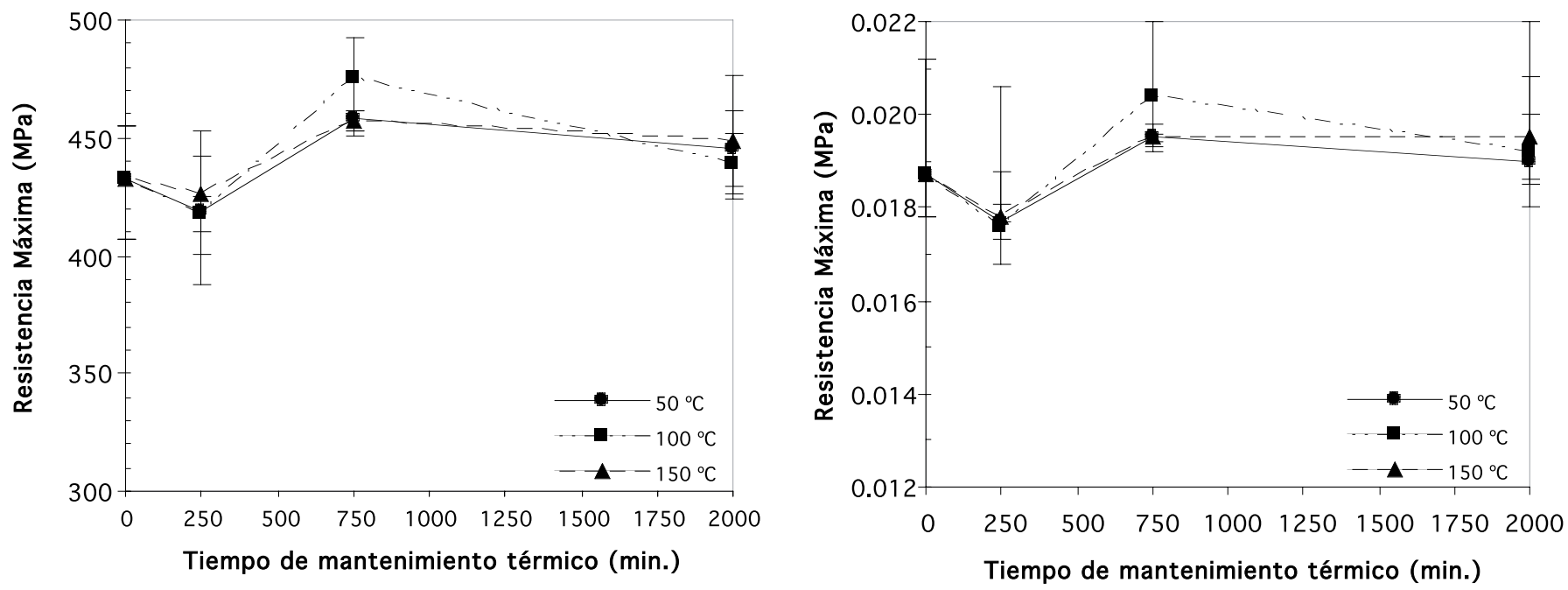

Figura 4: Resistencias y deformaciones vs. tiempo para las probetas envejecidas 30 días.

\subsection{Ensayos de termomecánicos de probetas envejecidas}

En las fig. 4 se representan los resultados de los ensayos termomecánicos realizados a las probetas envejecidas en la cámara higrotérmica, bajo las condiciones antes señaladas.

En estos resultados se observa una disminución en la resistencia a la tracción para las tres temperaturas en las probetas mantenidas en los primeros 250 minutos, situada entre el 1,7 y el 3,7 \%; luego un incremento entre el 5,3 y el $8,9 \%$ en los tiempos de mantenimiento de 750 minutos; y una disminución en las resistencias de las probetas que han permanecido 2000 minutos, entre el 1,4 y el 3,7\%; pero en este caso con valores superiores a los obtenidos con las probetas no mantenidas térmicamente. Para las probetas envejecidas, los resultados obtenidos con las deformaciones máximas de rotura también presentan un comportamiento similar al conseguido con las resistencias.

\section{CONCLUSIONES}

Se aprecia que el sometimiento de este tipo de material a una solicitación combinada de temperatura y tensión provoca una disminución en las propiedades mecánicas (resistencia y deformación última a tracción), sin que parezca que existe influencia de la temperatura. Las probetas envejecidas higrotérmicamente durante 30 días, al someterlas a los ensayos de mantenimiento térmico descritos en el presente trabajo, pierden propiedades durante cortos periodos de mantenimiento, pero luego se incrementan, alcanzando valores máximos de resistencia y deformación máxima de rotura en torno a los 750 minutos de ensayo. En este caso tampoco se observa una influencia de la temperatura empleada en los ensayos termomecánicos.

\section{BIBLIOGRAFÍA}

1. R. O. Bathgate, C. H. Wang, F. Pang, "Effects of temperature on the creep behavior of woven and stitched composites". Comp. Struct., 38 [1-4] 435-445 (1997).

2. F. Pang, C. H. Wang, R. G. Bathgate "Creep response of woven-fiber composites and the effect of stitching". Comp. Sci. \& Tech., 57 91-98 (1997).

3. B. D. Park, J. J. Balatinecz, "Short term flexural creep behavior of Woodfiber/polypropylene composites". Pol. Comp., 19 [4] 377-382 (1998).

4. J. Viña, M. Castrillo, A. Argüelles, I. Viña, “A comparison between the static and fatigue properties of glass-fiber and carbon-fiber reinforced polyetherimide composites after prolonged aging", Pol. Comp., 23, [4] 619-623 (2002).

5. K. Cheng K., K. Yoshioka, J. Seferis, "Hygrothermal cyclic effects on the durability of phenolic-based composites". Pol. Comp., 23, (2), 141-151, (2002).

6. B. Blackman, J. G. Williams, "Impact and High Rate Testing of Composites: An Overview", pp. 215-305, en NATO Adv. Study Inst. on Mech. of Comp. Mat. and Structures, (1999).

Recibido: 1.2.03

Aceptado: 30.11 .03 